Boletín de la Sociedad Geológica Mexicana

VOLUMEN 67, NÚM. 2, 2015, P. 203-214

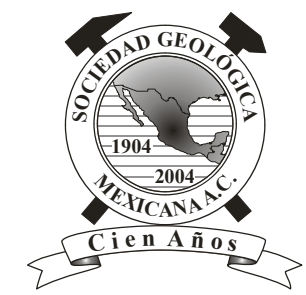

\title{
Estimación de parámetros mediante inversión y análisis de las pérdidas hidráulicas lineales y no-lineales durante el desarrollo y aforo del pozo San Lorenzo Tezonco
}

\author{
Eric Morales-Casique ${ }^{1, *}$, Oscar A. Escolero ${ }^{1}$, José L. Arce ${ }^{1}$ \\ ${ }^{1}$ Instituto de Geología, Universidad Nacional Autónoma de México, Ciudad Universitaria, 04510, México, D.F. \\ *ericmc@geologia.unam.mx
}

\begin{abstract}
Resumen
Se presenta un análisis de las pruebas de bombeo escalonadas en el pozo San Lorenzo Tezonco (SLT) localizado en la Ciudad de México y que alcanzó $2008 \mathrm{~m}$ de profundidad. Los parámetros hidrogeológicos y los parámetros correspondientes a las pérdidas de carga hidráulica debidas al pozo son estimados mediante inversión. Los resultados de las pruebas ilustran claramente el efecto del desarrollo mediante el bombeo donde la eficiencia del pozo se incrementa durante el desarrollo y durante las pruebas de aforo posteriores. Por otra parte, los valores de conductividad hidráulica y almacenamiento específico obtenidos en este trabajo son los primeros valores reportados en la literatura para la Cuenca de México correspondientes a las formaciones profundas entre 1176 y $2008 \mathrm{~m}$ de profundidad y que son representativos de una escala de cientos de metros. Estos valores pueden ser utilizados como parámetros iniciales en la calibración de modelos regionales de flujo de agua subterránea en la Cuenca de México. Adicionalmente, los parámetros del acuífero y del pozo SLT proporcionan valores de referencia útiles para planear aforos y el diseño constructivo de pozos de condiciones similares.

Palabras clave: Ciudad de México, hidrogeología, formaciones profundas, eficiencia hidráulica, estimación de parámetros, prueba de bombeo escalonada.

Abstract

We present an analysis of step-drawdown tests conducted at the San Lorenzo Tezonco (SLT) deep well located in Mexico City that reached a depth of $2008 \mathrm{~m}$. The hydrogeologic and well parameters are estimated through inversion. The results of the tests illustrate clearly the effect of well development through pumping, where the well efficiency increases during well-development and through successive step-drawdown tests. On the other hand, the values of hydraulic conductivity and specific storage obtained in this work are the first reported in the literature for the Basin of Mexico that correspond to the deep formations between 1176 and $2008 \mathrm{~m}$ and that are representative of a scale of hundreds of meters. These values can be used as initial values in the calibration of regional groundwater flow models in the Basin of Mexico. In addition, the values of hydrogeologic and well parameters obtained in this work are useful as reference values to plan and design future wells with similar conditions to the SLT well.
\end{abstract}

Keywords: Mexico City, hydrogeology, deep formations, hydraulic efficiency, parameter estimation, step drawdown test. 


\section{Introducción}

El estudio del sistema hidrogeológico de la Cuenca de México requiere el conocimiento de los parámetros que condicionan el flujo de agua subterránea. Específicamente se requiere conocer la conductividad hidráulica $K$ y el almacenamiento específico $S_{s}$ de los materiales geológicos. Para el acuífero en explotación de la Ciudad de México (aproximadamente hasta $500 \mathrm{~m}$ de profundidad), estos parámetros han sido obtenidos mediante pruebas de bombeo. Sin embargo, información sobre $K$ y $S_{s}$ correspondiente a profundidades mayores a $500 \mathrm{~m}$, es casi inexistente. P. Birkle y colaboradores [resultados no publicados, citados por Tinoco-Michel (2007)] obtuvieron valores de permeabilidad $k$ (también llamada permeabilidad intrínseca) mediante pruebas de laboratorio en núcleos provenientes de los pozos exploratorios perforados por PEMEX en la Cuenca de México (Pérez-Cruz, 1988). Aun cuando estos valores proporcionan una referencia valiosa a la escala de decenas de centímetros, el análisis y los modelos de simulación computacional de flujo de agua subterránea a escala regional requieren de parámetros representativos de escalas nominales de metros, decenas de metros, o incluso escalas mayores. Es por esto que a escala regional se necesitan parámetros estimados mediante pruebas de bombeo en pozos, los cuales típicamente corresponden a una escala nominal (estimada como la longitud del cedazo del pozo de bombeo) de decenas o incluso cientos de metros.

El pozo San Lorenzo Tezonco (SLT) fue perforado por el Sistema de Aguas de la Ciudad de México (SACMEX) y alcanzó $2008 \mathrm{~m}$ de profundidad. Este pozo ha proporcionado una oportunidad única para conocer la estratigrafía (Arce et al., 2013) y la hidrogeología (Morales-Casique et al., 2014) de las formaciones profundas de la Cuenca de México. Con base en el análisis del aforo del pozo SLT, MoralesCasique et al. (2014) reportaron los primeros valores $K$ y $S_{s}$ para la Cuenca de México correspondientes a una escala nominal de centenas de metros y de profundidad entre 1176 y 2008 m. En este trabajo el desarrollo y las pruebas de aforo en el pozo SLT son analizados para (a) reestimar los parámetros $K$ y $S_{s}$ mediante inversión considerando pérdidas de carga lineales y el efecto del volumen de agua almacenado dentro del pozo (factores no considerados anteriormente), (b) ilustrar cómo se incrementa la eficiencia de un pozo con el desarrollo y que la mejora en la eficiencia continúa con su operación durante los aforos, (c) discutir cómo un aforo debe probar el acuífero sin verse limitado por equipo de bombeo inadecuado, (d) discutir cómo los valores estimados de los parámetros pueden ser utilizados para un diseño preliminar de futuras captaciones a tales profundidades, y (e) discutir cómo la selección del caudal de operación debe considerar no sólo la eficiencia hidráulica sino también efectos como turbulencia, velocidad de entrada del agua al ademe ranurado y el incremento de las pérdidas de carga hidráulica de pozo.

\section{Métodos}

Los parámetros $K$ y $S_{s}$ típicamente son obtenidos mediante pruebas de bombeo a caudal constante en pozos en producción, registrando el abatimiento en el pozo de bombeo y en uno o más pozos de observación (Kruseman y De Rider, 1994; Batu, 1998). Alternativamente, las pruebas de bombeo de caudal escalonado consisten en extraer agua de un pozo a un caudal constante hasta que un estado cuasiestacionario en el abatimiento ha sido observado. El caudal de extracción es entonces incrementado hasta que un nuevo estado cuasi-estacionario es alcanzado y el procedimiento se repite para caudales adicionales. Estas pruebas de caudal escalonado (step-drawdown tests), también conocidas como aforos, permiten estimar los parámetros $K$ y $S_{s}$ del acuífero $\mathrm{y}$, además, evaluar las pérdidas de carga no lineales que son necesarias para la selección del caudal de operación del pozo (Rorabaugh, 1953; Avci, 1992; Kruseman y De Rider, 1994; Kawecki, 1995; Batu, 1998; Jha et al., 2006; Shekhar, 2006; Louwyck et al., 2010; Mathias y Todman, 2010; Avci et al., 2010).

El flujo radial horizontal de agua subterránea hacia un pozo en un acuífero confinado es gobernado por la ecuación

$$
\frac{1}{r} \frac{\partial}{\partial r}\left(b K_{r} r \frac{\partial s}{\partial r}\right)=S_{s} b \frac{\partial s}{\partial t}
$$

donde $b$ es el espesor saturado del acuífero, $s(r, t)=h_{0}-h(r, t)$ es abatimiento, $h_{0}$ es la carga hidráulica al tiempo $t=0, h$ es la carga hidráulica después de iniciado el bombeo, $r$ es distancia radial, $K_{r}$ es conductividad hidráulica radial $[L / T]$ y $S_{s}$ es almacenamiento específico $[1 / L]$. La ecuación (1) debe ser sujeta a condiciones de frontera apropiadas. Para un caudal de bombeo $Q$ constante $\left[L^{3} / T\right]$ la solución de (1) se puede expresar como

$$
s(r, t)=B_{1}(r, t) Q
$$

donde $B_{1}(r, t)=W(u) / 4 \pi K_{r} b$ y $W(u)=\int_{u}^{\infty} \frac{e^{-y}}{y} d y$ es la función de pozo de Theis, con $u=r^{2} S_{s} / 4 K_{r} t$. En un pozo de bombeo ideal (de radio cero y que su construcción no alteró el acuífero), el abatimiento estaría dado por (2) y expresa las pérdidas de carga en el acuífero donde el flujo de agua subterránea obedece la ley de Darcy; estas pérdidas de carga dependen linealmente del caudal de bombeo. Sin embargo, existen pérdidas de carga adicionales causadas por el pozo de bombeo y por el régimen de flujo no Darciano que se desarrolla dentro del acuífero en la vecindad del pozo (Jacob, 1947; Rorabaugh, 1953; Şen, 1988; Camacho-V. y Vásquez-C., 1992; Pérez-Franco, 1994; Pérez-Franco, 1996; Mathias et al., 2008; Wen et al., 2008; Mathias y Todman, 2010). El régimen no Darciano en el acuífero implica una relación no lineal con la descarga específica. Una de las expresiones más utilizadas es la propuesta por Forchheimer (1901) 


$$
\frac{\partial s}{\partial r}=\alpha q_{r}+\beta q_{r}^{2}
$$

donde $\alpha$ y $\beta$ son constantes y $q_{r}$ es la descarga específica $[L / T]$ en la dirección radial (caudal dividido por área transversal unitaria). Obsérvese que cuando $\beta=0$ se recupera la ley de Darcy con $\alpha=1 / K_{r}$. Con base en la ecuación (3) y utilizando el método de expansión asintótica empalmada, Mathias et al. (2008) desarrollaron una expresión para el abatimiento en el pozo de bombeo que considera el régimen no Darciano

$$
\left.s(t)\right|_{r_{w}}=c_{1}(t) Q+c_{2} Q^{2}
$$

donde $r_{w}$ es el radio del pozo, $c_{1}(t)=\frac{1}{4 \pi K_{r} b}\left[\ln \left(4 K_{r} t / S_{s} r_{w}^{2}\right)-0.5772\right]$ corresponde a la aproximación de Cooper-Jacob para tiempos grandes y $c_{2}=\beta /(2 \pi b)^{2} r_{w}$. Pérez-Franco (1996) propuso una expresión similar a (4) pero donde $c_{1}(r, t)=B_{1}(r, t)$ de la ecuación (2). Alternativamente se puede resolver numéricamente la ecuación de flujo considerando (3) en lugar de la ley de Darcy (Mathias y Todman, 2010) o dividiendo el dominio de simulación en dos zonas, una de flujo no Darciano y otra exterior de flujo Darciano (Wen et al., 2008). En este trabajo utilizaremos una expresión similar a la ecuación (4).

Las pérdidas de carga debidas al pozo de bombeo tienen dos componentes: una lineal y otra no-lineal. Las pérdidas lineales de carga en la vecindad del pozo son debidas a compactación del acuífero durante la perforación, invasión del lodo de perforación y pérdidas de carga en el filtro de grava y el cedazo; las pérdidas no-lineales comprenden pérdidas por fricción debidas a flujo turbulento dentro del cedazo y el interior del pozo (Barker y Herbert, 1992). Las pérdidas de pozo se expresan como (Rorabaugh, 1953)

$$
s_{p}(t)=B_{2}(t) Q+C Q^{n}
$$

donde $B_{2}$ es un coeficiente con unidades $T / L^{2}, C$ es otro coeficiente con unidades $T^{n} / L^{3 n-1}$ y $n$ es un exponente que ha sido propuesto igual a 2 (Jacob, 1947) o de manera más general es ajustado de acuerdo a los datos de campo de la prueba escalonada (Rorabaugh, 1953). Si se desprecia el almacenamiento de la zona alterada vecina al pozo (equivalente a suponer flujo en estado estacionario en esa zona), entonces las pérdidas lineales debidas al pozo se pueden expresar en forma de un abatimiento constante en el tiempo y proporcional al caudal (Rushton, 2003)

$$
B_{2}(t) \approx B_{2}=\frac{S_{w}}{2 \pi K_{r} b}
$$

donde $S_{w}$ es conocido como factor de "piel" (skin factor), adimensional.

Con base en las ecuaciones (4) y (5), en este trabajo adoptamos el supuesto de que las pérdidas hidráulicas no lineales debidas a flujo no Darciano en la vecindad del pozo y a flujo turbulento en el pozo de bombeo pueden ser descritas conjuntamente por el término $C Q^{n}$. Combinando (2), (5) y (6), el abatimiento total en el pozo de bombeo es

$$
s_{\text {bom }}(t)=s+s_{p}=B_{1}\left(r_{w}, t\right) Q+\frac{S_{w}}{2 \pi K_{r} b} Q+C Q^{n}
$$

donde $r_{w}$ es el radio del pozo de bombeo.

En este trabajo analizamos la evolución de las pérdidas de carga no atribuibles a flujo Darciano en el acuífero, denotadas por $s_{p}$ en la ecuación (7), y de la eficiencia para ilustrar la efectividad del desarrollo y que el proceso de desarrollo (disminución de $s_{p}$ ) continúa durante aforos posteriores. La eficiencia de un pozo se define como la razón de las pérdidas de carga lineales en el acuífero y las pérdidas de carga totales (Kruseman y De Rider, 1994; Kawecki, 1995)

$$
E_{w}=100 \frac{s}{s+s_{p}}
$$

Estrictamente $s$ debiera incluir las pérdidas de carga debidas a flujo no Darciano en el acuífero; sin embargo, con los datos recabados no fue posible separarlas de las pérdidas no lineales debidas al pozo. En el caso ideal, si $s_{p}=0$ entonces el pozo tiene una eficiencia del $100 \%$. La eficiencia de un pozo es un concepto útil para identificar cuando se necesita dar mantenimiento a un pozo (Peña-Díaz y ArreguínMañón, 1989). En este trabajo el concepto de eficiencia se utiliza para ilustrar el proceso de desarrollo del pozo. Sin embargo, cabe aclarar que el concepto de eficiencia no es particularmente útil para comparar pozos (Kawecki, 1995). Por ejemplo, supongamos que dos pozos son construidos de manera idéntica tal que $s_{p}$ es igual en ambos, pero uno está en una zona menos permeable del acuífero. Para un mismo abatimiento $s$, ambos pozos tendrán la misma eficiencia pero el pozo en la zona menos permeable producirá un menor caudal; en este sentido, utilizar la ecuación (8) para comparar pozos puede conducir a interpretaciones erróneas.

\subsection{Solución numérica}

La ecuación (1), sujeta a condiciones de frontera apropiadas, puede ser resuelta mediante métodos numéricos. Rathod y Rushton (1991) propusieron un modelo conceptual de dos capas, en el que en cada capa el flujo es radial gobernado por la ecuación (1) suplementada con términos fuente para representar el flujo vertical entre capas a través de una capa de baja permeabilidad (acuitardo) en la que se desprecia el efecto del almacenamiento; las ecuaciones resultantes son resueltas mediante diferencias finitas. Para discretizar la ecuación (1) en la dirección radial se introduce el cambio de variable $a=1 \mathrm{n} r$, de manera que un incremento uniforme $\Delta a$ corresponde a un incremento logarítmico en la coordenada radial $r$. La dirección radial es discretizada en seis incrementos $\Delta a$ por cada incremento en orden de magnitud de $r$; por ejemplo, el intervalo $r_{w} \leq r \leq 10 r_{w}$ es discretizado en seis elementos, donde la longitud de cada elemento se incrementa logarítmicamente. También el tiempo de simulación es discretizado logarítmicamente, aunque utilizando una discretización más fina de diez pasos de tiempo por cada orden de magnitud de incremento en el 
tiempo. La discretización adoptada en $r$ y $t$ es suficiente para reproducir con exactitud razonable la solución analítica de Theis (Rushton, 2003). La ecuación (1) es aproximada en diferencias finitas por (Rushton, 2003)

$\frac{1}{\Delta a}\left(b K_{r, n-1} \frac{s_{n-1}^{t+\Delta t}-s_{n}^{t+\Delta t}}{\Delta a}-b K_{r, n} \frac{s_{n}^{t+\Delta t}-s_{n+1}^{t+\Delta t}}{\Delta a}\right)=\frac{S_{s, n} b r_{n}^{2}}{\Delta t}\left(s_{n}^{t+\Delta t}-s_{n}^{t}\right)+q_{n} r_{n}^{2}$

donde $s_{n}^{t+\Delta t}=s\left(r_{n}, t+\Delta t\right)$ y $K_{r, n-1}$ es $K_{r}$ interpolada entre los nodos $n-1$ y $n$. Detalles adicionales del método numérico y el código computacional están disponibles en Rathod y Rushton (1991) y en Rushton (2003). El código computacional de Rathod y Rushton (1991) resuelve (9) y proporciona el primer término de la ecuación (7). Permite además incluir el efecto del agua almacenada dentro del pozo (wellbore storage) extendiendo la discretización al interior del pozo y asignando a esas celdas una conductividad hidráulica alta y un coeficiente de almacenamiento igual a 1; esto proporciona el segundo término de la ecuación (7). Para analizar pruebas escalonadas, en este trabajo modificamos el código computacional para añadir el término $C Q^{n}$, completando el cálculo de (7).

\subsection{Problema inverso}

La inversión de una prueba escalonada se centra en determinar los parámetros que reproducen el abatimiento en el pozo de bombeo: los parámetros del acuífero $K_{r}$ y $S_{s}$, el parámetro de las pérdidas lineales en la vecindad del pozo $S_{w}$, y los parámetros de las pérdidas no lineales $C$ y $n$. Para la inversión se utilizó el código PEST (Doherty, 2002). PEST es una herramienta general independiente del modelo a calibrar (es decir, no se necesita modificar el código del modelo), implementa un método de regresión no lineal generalizada y proporciona herramientas para examinar la sensibilidad del modelo a cada parámetro.

\subsection{El pozo San Lorenzo Tezonco}

El pozo San Lorenzo Tezonco (SLT) se localiza en las coordenadas $19^{\circ} 22^{\prime} 44.27^{\prime \prime} \mathrm{N}$ y $99^{\circ} 5^{\prime}$ 5.25" O (Figura 1). Su perforación fue conducida en tres etapas: (a) de 0 a 500 $\mathrm{m}$ de profundidad, (b) de 500 a $1140 \mathrm{~m}$ de profundidad, y (c) de 1140 a $2008 \mathrm{~m}$ de profundidad. Para detalles adicionales sobre la perforación en cada etapa se puede consultar Morales-Casique et al. (2014). La Figura 2 muestra el corte litológico, el diseño constructivo del pozo SLT y el modelo hidrogeológico conceptual adaptado al modelo de dos acuíferos de Rathod y Rushton (1991). El ademe ranurado se encuentra de 1176 a $2008 \mathrm{~m}$ de profundidad y en esa longitud el pozo fue terminado sin filtro de grava (Morales-Casique et al., 2014). Al concluir la perforación, el pozo fue desarrollado mediante bombeo por etapas, aumentando el caudal durante 71 horas. El objetivo del desarrollo es maximizar la capacidad específica (caudal / abatimiento) del pozo; en particular se busca no solo restaurar las propiedades hidráulicas naturales de la formación y que fueron degradadas por la invasión de los fluidos de perforación (típicamente a base de bentonita) sino en lo posible incrementar esas propiedades (mediante la extracción de la fracción fina) en la porción de la formación adyacente al pozo. Posterior al desarrollo, se condujo el aforo con el objetivo de determinar el caudal explotable del pozo en función de sus características hidráulicas.

El corte litológico mostrado en la Figura 2 se basa en el trabajo de Arce et al. (2013) y actualizado en Arce et al. (2015), en este número. Con base en el corte litológico pero sobre todo en el registro eléctrico, Morales-Casique et al. (2014) proponen cinco unidades hidrogeológicas (Figura 2). En este trabajo, para el modelo de dos capas de Rathod y Rushton (1991), los acuíferos 1 y 2 (Figura 2) son agrupados en una sola unidad. Puesto que no se cuenta con mediciones de carga hidráulica dentro del acuitardo que sobreyace al acuífero 3, no es posible estimar sus propiedades en respuesta a las pruebas hidráulicas conducidas; en este trabajo se le asigna una $K$ baja igual a $1 \times 10^{-6} \mathrm{~m} /$ día. La frontera inferior del acuífero 3 se supone impermeable, por lo que el acuífero 3 es modelado como confinado para efectos prácticos. Adicionalmente, el registro eléctrico (Figura 2) muestra que estas unidades, y en particular el acuífero 3 ubicado de 1140 a 2008 m, son heterogéneas, por lo que Morales-Casique et al. (2014) interpretan los parámetros $K$ y $S_{s}$ en (1) como parámetros promedio definidos por

$$
\begin{aligned}
& \bar{K}=\frac{1}{b} \int_{0}^{b} K(z) d z \\
& \overline{S_{s}}=\frac{1}{b} \int_{0}^{b} S_{s}(z) d z
\end{aligned}
$$

donde $K(z)$ y $S_{s}(z)$ son la conductividad hidráulica y el almacenamiento específico a la profundidad $z$. Esta definición de parámetros efectivos es adoptada en este trabajo.

\section{Resultados}

El desarrollo del pozo tuvo una duración de 71 horas. Concluido el desarrollo, el nivel se dejó recuperar durante 1 hora (aunque el tiempo no fue suficiente para una recuperación total) y se condujo el primer aforo (aforo 1) que duró 35 horas y consistió en 6 etapas. 12 horas después del aforo 1 se condujo un segundo aforo (aforo 2). Para el aforo 3 , conducido 15 días después del aforo 2 , se consiguió un equipo con mayor capacidad de bombeo. Durante las primeras tres pruebas (desarrollo, aforos $1 \mathrm{y}$ 2) se colectaron muestras de agua para su análisis físicoquímico y se registró de manera periódica temperatura y conductividad eléctrica del agua a la descarga del pozo con el fin de tener un control sobre la evacuación del agua ajena a la formación (por la limpieza) y la representatividad de las muestras de agua. Durante el aforo 3 solo se colectaron 


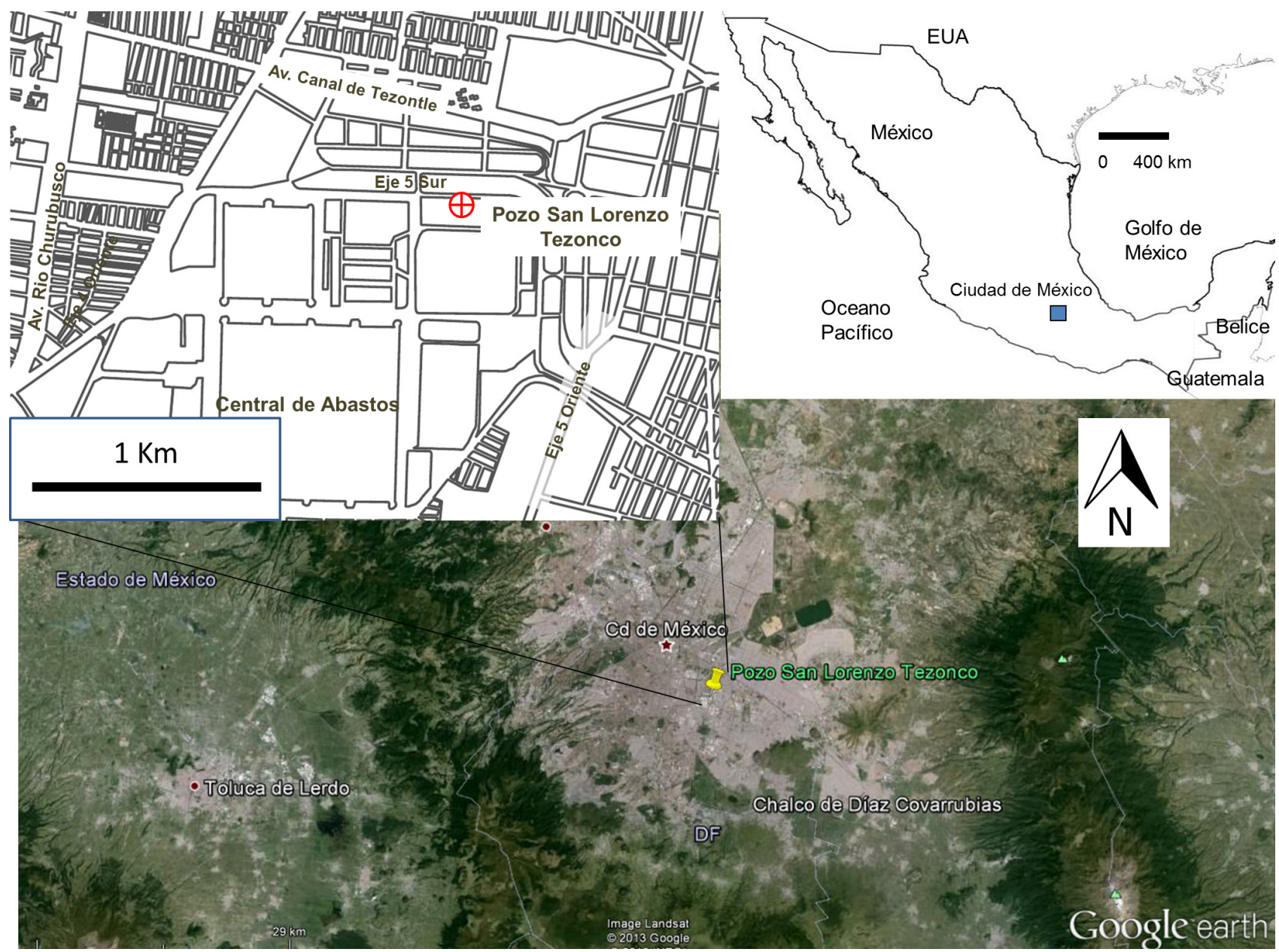

Figura 1. Ubicación del pozo San Lorenzo Tezonco.

datos de temperatura. Los datos disponibles del desarrollo y los aforos del pozo SLT se muestran en la Figura 3. Una discusión sobre los cambios en temperatura y conductividad eléctrica observados durante el bombeo y su interpretación hidrogeológica se puede consultar en Morales-Casique et al. (2014).

\subsection{Inversión del aforo}

La Figura 4 muestra el ajuste obtenido mediante inversión entre el abatimiento medido y el observado durante las diez etapas del aforo 3 , así como la variación de la temperatura medida a la descarga del pozo. Es interesante notar que el modelo sobreestima el abatimiento en las primeras dos etapas ( $<1$ día) y en la etapa 8 (alrededor de 5 días) mientras que subestima el abatimiento en la sexta etapa (entre 3 y 4 días). El comportamiento anómalo durante la etapa 8 sugiere que los datos contienen errores o que existieron variaciones en el caudal de extracción que no fueron detectadas. Por otra parte, el proceso de inversión finalizó con una solución intermedia entre las etapas 1 y 2 y la etapa 6; en las etapas 1 y 2 los valores modelados son mayores a los observados mientras que en la etapa 6 los valores modelados son menores a los observados, por lo que los parámetros ajustados logran un compromiso para minimizar estas diferencias. Un factor adicional que puede influir en la interpretación de las primeras etapas del aforo 3 es la variación de la temperatura. La viscosidad del agua a $50{ }^{\circ} \mathrm{C}$ es aproximadamente el $90 \%$ de la viscosidad a $45^{\circ} \mathrm{C}$, por lo que la relación entre las conductividades hidráulicas a tales temperaturas será $K_{45^{\circ} \mathrm{C}}=0.9 K_{50^{\circ} \mathrm{C}}$ (despreciando los cambios de densidad). Sin embargo, para investigar estos efectos en trabajos futuros será necesario resolver la ecuación de transferencia de calor acoplada a la ecuación de flujo (1).

Los parámetros estimados mediante inversión se muestran en la Figura 5, junto con los correspondientes intervalos de confianza (al $95 \%$ de probabilidad) centrados en el valor estimado. Un intervalo de confianza proporciona el rango dentro del cual es probable que se encuentren los parámetros desconocidos. El hecho de que los intervalos de confianza en la Figura 5 son relativamente pequeños indica que la incertidumbre en los parámetros estimados es pequeña. Cabe señalar que los valores obtenidos de los 
a)

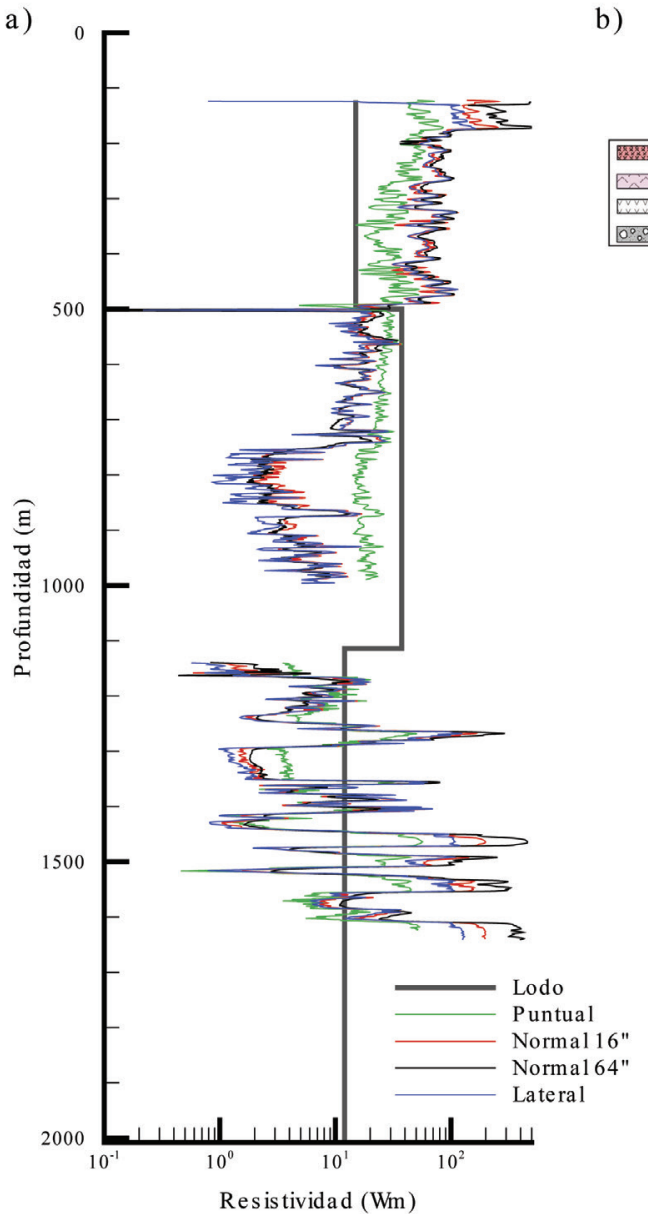

b)
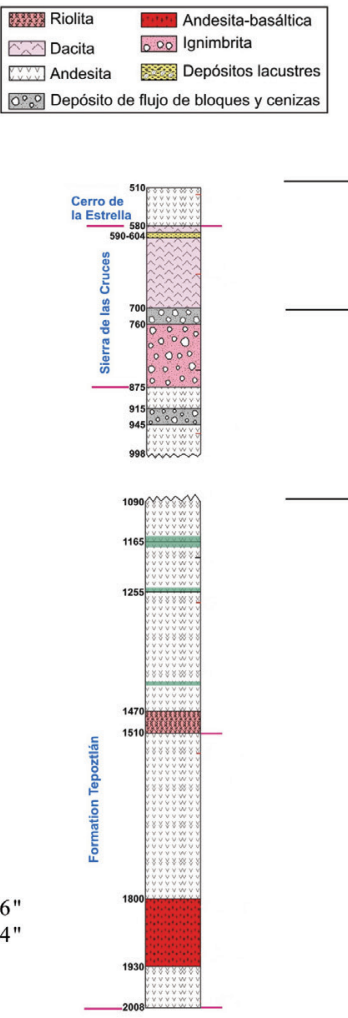

c)

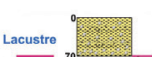

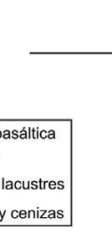
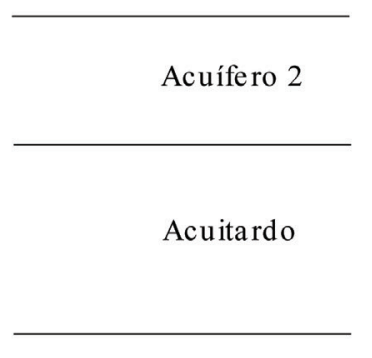

Acuífero 1

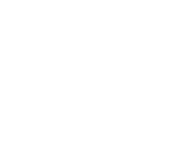

Acuífe ro 3 d)
Ademe lis o cédula 40, con diámetro de $0.323 \mathrm{~m}$ $(12 " 3 / 4)$, espesor de $0.95 \mathrm{~cm}\left(3 / 8^{\prime \prime}\right)$

Figura 2. Datos del pozo San Lorenzo Tezonco: a) registro eléctrico, b) corte litológico (adaptado de Arce et al., 2015), c) modelo conceptual hidrogeológico y d) esquema constructivo del pozo.

parámetros en este trabajo son similares a los obtenidos en Morales-Casique et al. (2014), aun cuando estos autores despreciaron el efecto del agua almacenada dentro del pozo de bombeo (Tabla 1). Esto es debido a que el efecto del volumen de agua, almacenado dentro de la perforación, sólo es significativo al inicio de cada etapa tal como se muestra en la Figura 4 y durante el proceso de inversión el ajuste se centra a los tiempos largos de cada etapa; adicionalmente, no se contó con datos de niveles a tiempos cortos en cada etapa.

La Tabla 2 contiene la matriz de correlación de los parámetros estimados. La mayor correlación existe entre los parámetros $C$ y $n(-0.97)$ y entre $S_{s}$ y $S_{w}(0.86)$. Correlaciones altas entre parámetros típicamente dificultan el proceso de inversión. Aun con esa correlación relativamente alta, los intervalos de confianza de los parámetros estimados están acotados y su magnitud es razonable (Figura 5). Finalmente, la estimación de $S_{s}$ debe tomarse con precaución porque solo se contó con datos de abatimiento en el pozo de bombeo. Por ejemplo, si se emplea la solución analítica de Theis o la Cooper-Jacob (Batu, 1998), una vez determinado el valor de $K_{r}$, el valor de $S_{s}$ se obtiene de expresiones que son proporcionales a $1 / r^{2}$, las cuales crecen sin límite conforme $r$ tiende a cero. Por lo tanto los datos del pozo de bombeo no son apropiados para calcular $S_{s}$. Sin embargo, el modelo numérico empleado en este trabajo se basa en una representación de radio finito para el pozo de bombeo e incluye factores adicionales como el efecto del almacenamiento de agua dentro del pozo, las pérdidas lineales debido a alteración de la formación alrededor del pozo y las pérdidas no lineales. Creemos que al considerar explícitamente estos factores se reduce la incertidumbre (aunque no la elimina) en la determinación de $S_{s}$ a partir de datos únicamente del pozo de bombeo.

\subsection{Evolución de las pérdidas de pozo}

Los parámetros del acuífero estimados en la sección anterior fueron utilizados para calcular las pérdidas de carga debidas al pozo y al régimen no Darciano en su vecindad, es decir, la diferencia entre el abatimiento observado menos el abatimiento lineal debido al acuífero, $s_{p}=s_{b o m}-s$ de la ecuación (6). Cabe enfatizar que $s_{p}$ incluye tanto pérdidas 

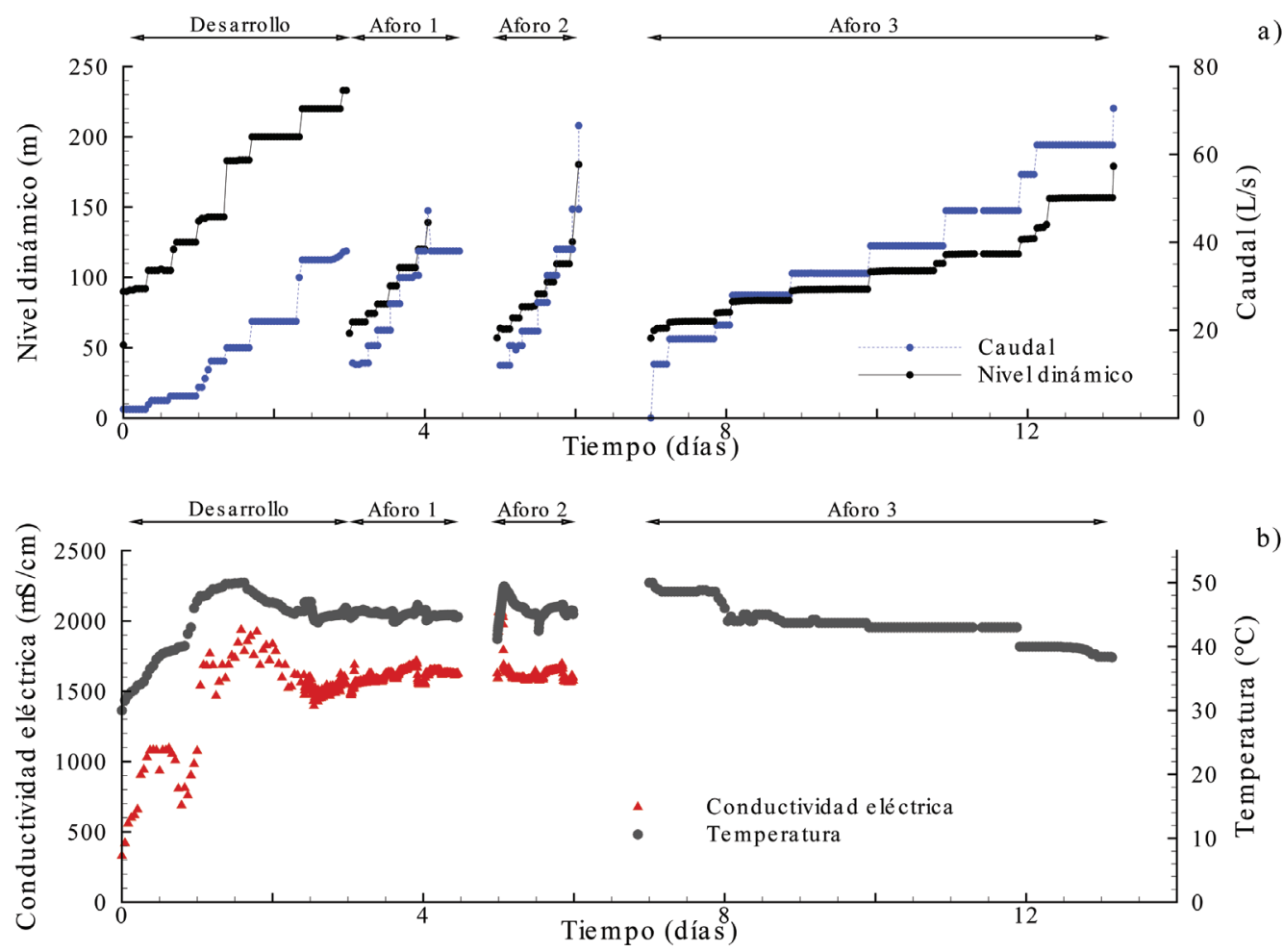

Figura 3. Desarrollo y aforos 1, 2 y 3 en el pozo San Lorenzo Tezonco. Para presentación gráfica el aforo 3 inicia en el día 7, pero en realidad se condujo 15 días después del final del aforo 2. (a) Nivel dinámico y caudal, (b) Temperatura y conductividad eléctrica.

a)

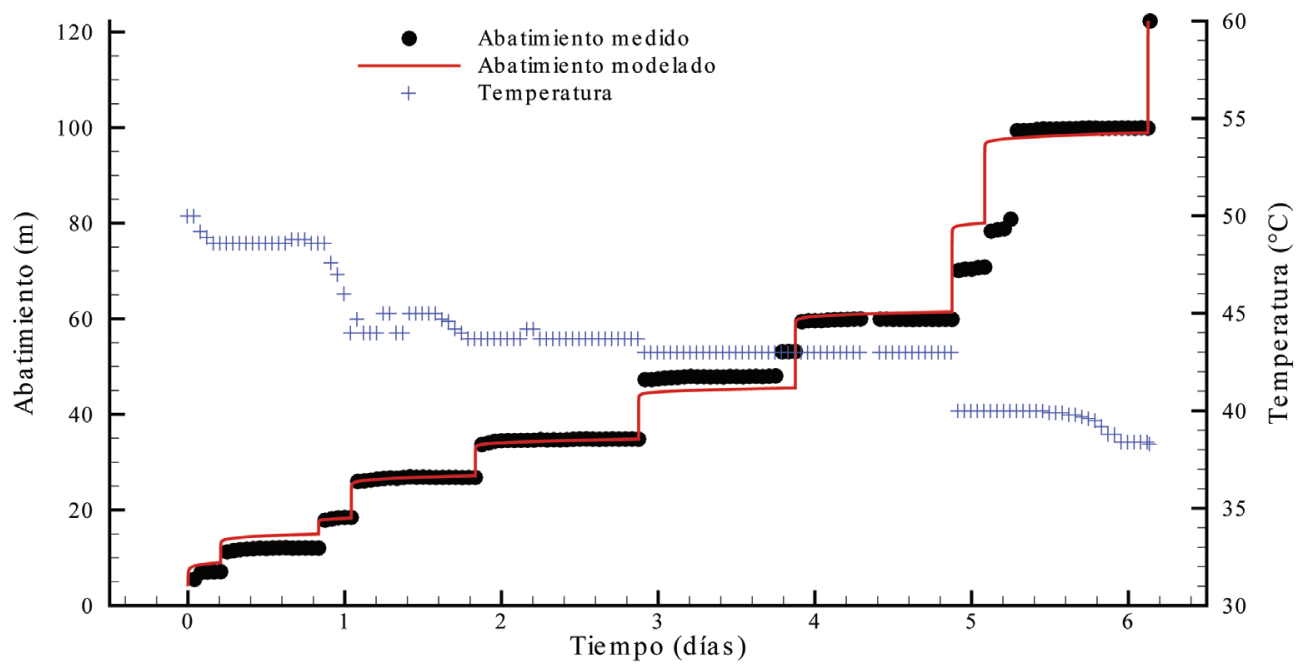

b)

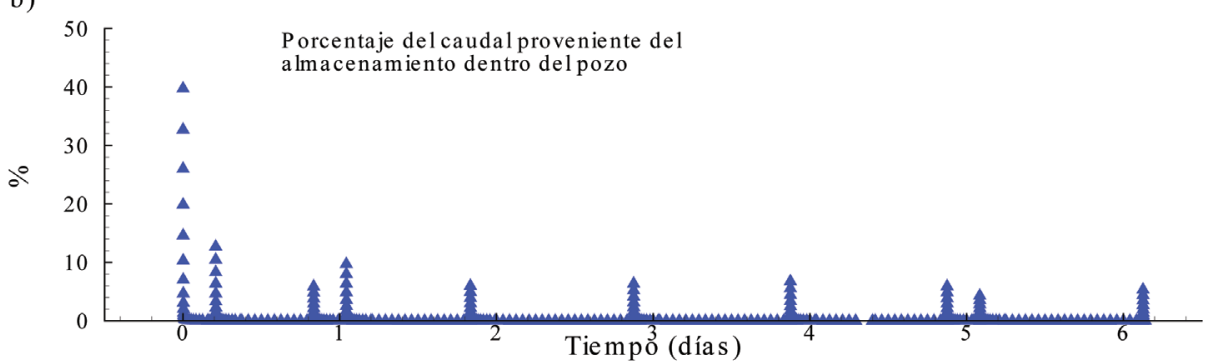

Figura 4. (a) Ajuste obtenido mediante inversión del aforo 3 y (b) porcentaje del caudal proveniente del almacenamiento dentro del pozo. 
Tabla 1. Resultados de la inversión del aforo 3.

\begin{tabular}{llll}
\hline Parámetro & Inversión & $\begin{array}{l}\text { Morales-Casique et al. } \\
(2014)\end{array}$ & Unidades \\
\hline$K_{r}$ & 0.245 & 0.2 & $\mathrm{~m} /$ día \\
$S_{s}$ & $1.15 \times 10^{-5}$ & $4 \times 10^{-6}$ & $1 / \mathrm{m}$ \\
$S_{w}$ & 1.086 & $\mathrm{NA}$ & adimensional \\
$C$ & $1.93 \times 10^{-8}$ & $3 \times 10^{-8}$ & $\mathrm{día}^{n} / \mathrm{m}^{3 n-1}$ \\
$n$ & 2.529 & 2.465 & adimensional $^{n}$ \\
\hline NA- No aplica & &
\end{tabular}

Tabla 2 Matriz de correlación (adimensional) de los parámetros estimados, aforo 3.

\begin{tabular}{cccccc}
\hline & $K_{r}$ & $S_{s}$ & $S_{w}$ & $C$ & $n$ \\
\hline$K_{r}$ & 1 & -0.23 & -0.034 & 0.016 & 0.19 \\
$S_{s}$ & -0.23 & 1 & 0.86 & 0.023 & -0.051 \\
$S_{w}$ & -0.034 & 0.86 & 1 & 0.11 & -0.12 \\
$C$ & 0.016 & 0.023 & 0.11 & 1 & -0.97 \\
$n$ & 0.19 & -0.051 & -0.12 & -0.97 & 1 \\
\hline
\end{tabular}

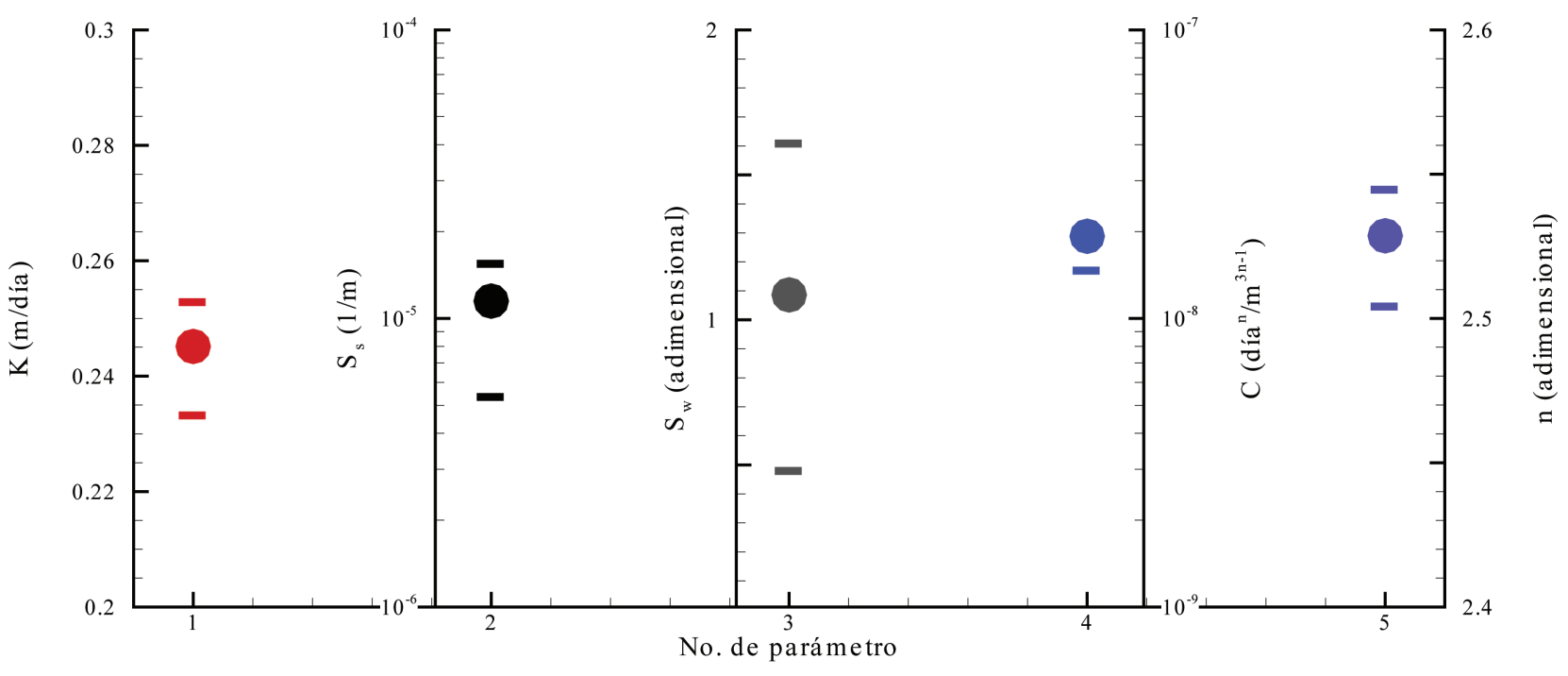

Figura 5. Parámetros estimados e intervalos de confianza ( $95 \%$ de probabilidad) obtenidos mediante la inversión del aforo 3.

de carga que dependen linealmente del caudal de extracción como pérdidas de carga no lineales dentro del pozo y aquellas debidas al régimen no Darciano. La magnitud de $s_{p}$ al final de cada etapa de bombeo escalonado se muestra en la Figura 6. En la misma figura las líneas continuas representan el abatimiento debido a pérdidas de carga lineales en el acuífero en cada prueba (desarrollo, aforo 1, 2, y 3). Las líneas continuas presentan quiebres debido a que cada etapa de la prueba escalonada tuvo duración diferente y por tanto el abatimiento debido al acuífero es diferente tal como lo indica la ecuación (2); si las etapas de las pruebas escalonadas tuvieran la misma duración entonces el abatimiento debido al acuífero formaría una sola línea recta.

De la Figura 6 es evidente que durante el desarrollo, las pérdidas de carga debido al pozo superan en magnitud al abatimiento debido al acuífero. Una vez concluida la séptima etapa del desarrollo se procedió al aforo 1 (Figura 3) donde se registra una disminución drástica en las pérdidas de carga debidas al pozo (Figura 6). Esto sugiere que un método de limpieza y desarrollo efectivo debiera alternar periodos de bombeo y periodos de reposo, o periodos donde se reduzca el gasto con periodos donde se incremente. Posteriormente, durante los aforos 2 y 3 el proceso de limpieza y desarrollo del pozo continúa, tal como se observa que $s_{p}$ disminuye del aforo 1 al 2, y del 2 al 3, para caudales de extracción similares (Figura 6). El hecho de que el modelo ajustado no reproduce adecuadamente las dos primeras etapas del aforo 3 se refleja en la existencia de valores negativos ( $\sin$ significado físico) de $s_{p}$ en la Figura 6 para la primera etapa de los aforos 1 y 2 .

La Figura 7 muestra la evolución de la eficiencia del pozo, definida por la ecuación (7), durante el desarrollo y los aforos 1, 2 y 3. Durante el desarrollo se observa un incremento en la eficiencia al aumentar el caudal de extracción; sin embargo, la eficiencia se mantiene menor al $20 \%$. Al cesar el bombeo e iniciarlo una hora después para efectuar el aforo 1, la eficiencia se incrementa de manera drástica, sugiriendo que alternar periodos de bombeo y recuperación puede tener un efecto benéfico en la limpieza y desarrollo del pozo y hacer más efectiva esta etapa. La Figura 7 ilustra que durante los aforos posteriores, para un mismo caudal, la eficiencia se incrementa, es decir, las pérdidas de carga debidas al pozo $s_{p}$ disminuyen. Es de 


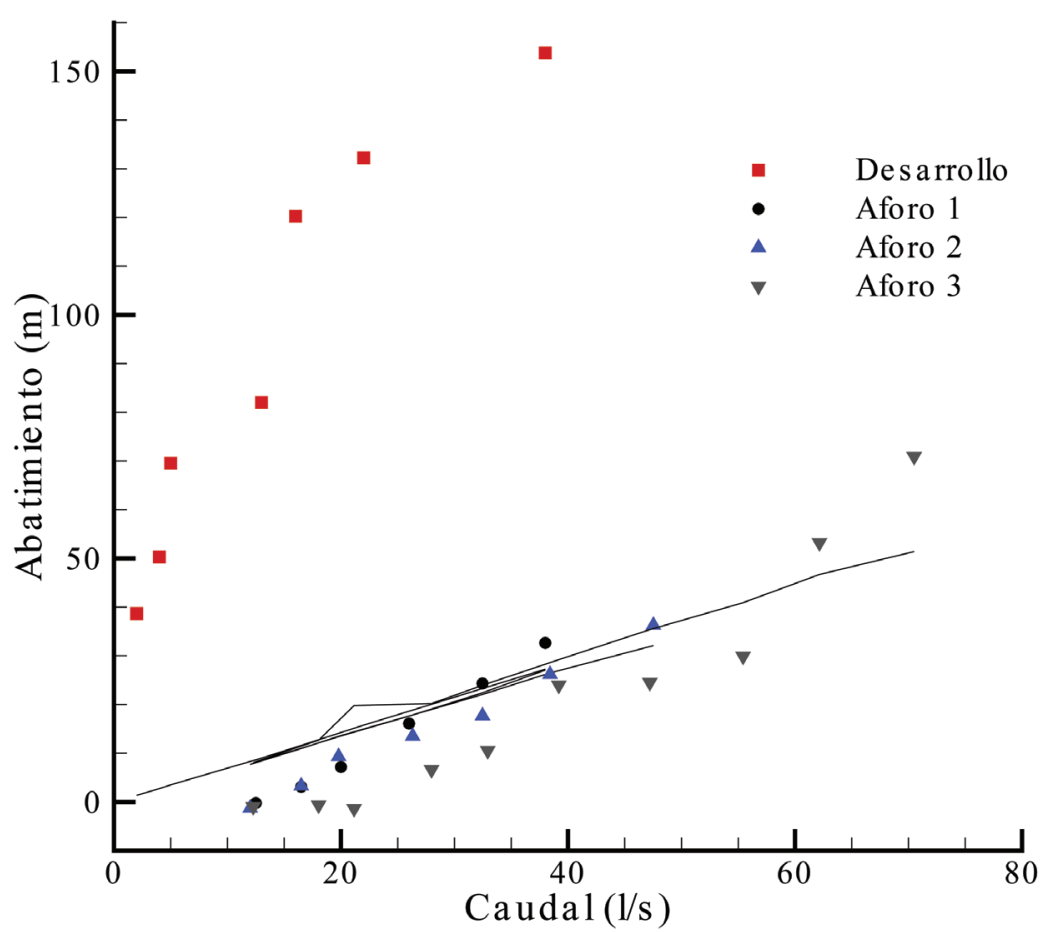

Figura 6. Evolución de la suma de las pérdidas de pozo y las pérdidas por flujo no Darciano durante el desarrollo y los aforos 1, 2 y 3.

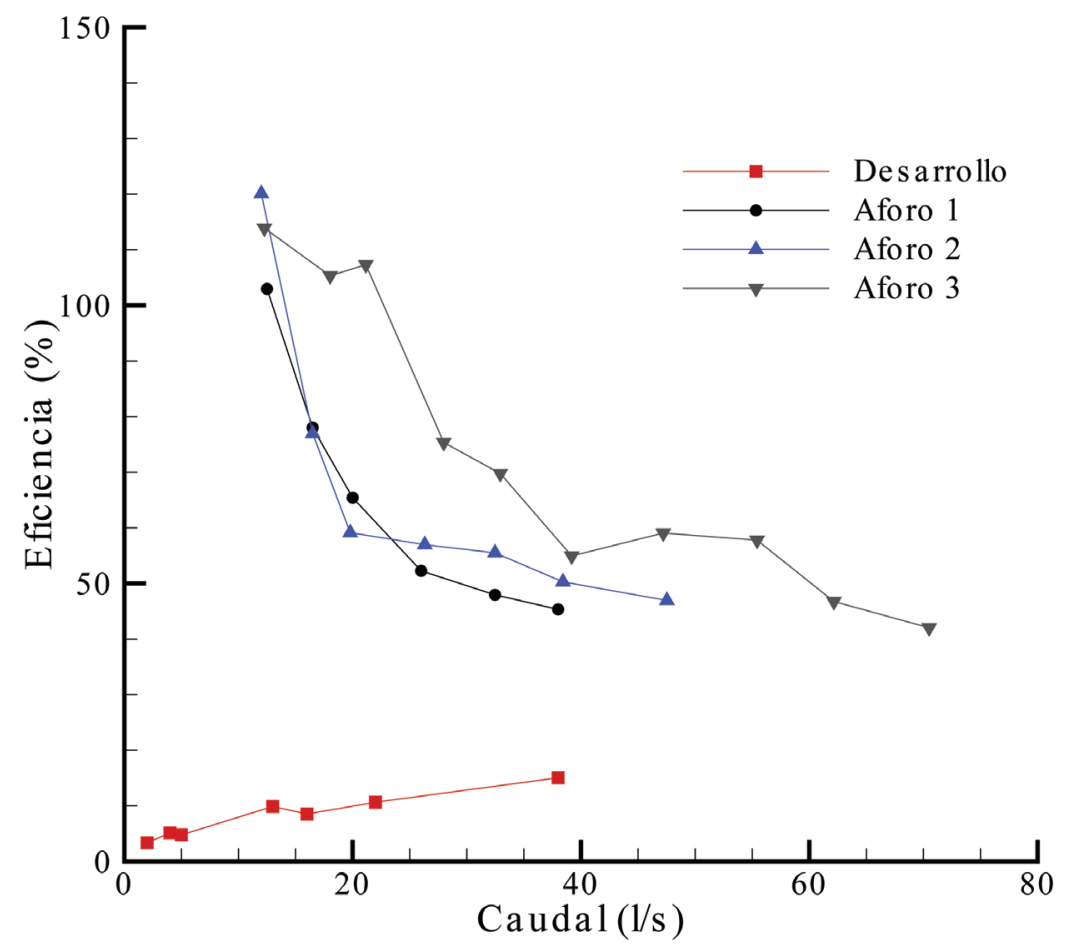

Figura 7. Evolución de la eficiencia durante el desarrollo y los aforos 1,2 y 3. 
esperar que durante la operación del pozo este incremento en la eficiencia continúe. Después de varios años de operación la eficiencia probablemente disminuirá debido a problemas como incrustación, azolve y otros.

Otro aspecto importante de la Figura 7 es la disminución de la eficiencia del pozo al aumentar el caudal de extracción. Alrededor de los 60 l/s la eficiencia registrada en el aforo 3 es del orden de $50 \%$, es decir, las pérdidas de carga debidas al pozo y al régimen no Darciano igualan en magnitud a las pérdidas lineales debidas al acuífero. Este es uno de los factores a considerar en la selección del caudal óptimo de operación de un pozo. La selección del caudal de operación debería incluir también un análisis costo-beneficio, ponderando el beneficio de obtener una mayor cantidad de agua comparado con el costo que implica extraerla a mayor profundidad.

Un aspecto adicional es el área abierta del ademe ranurado. Típicamente, el ademe ranurado se selecciona de manera que proporcione el área abierta necesaria para asegurar que, bajo el caudal de extracción deseado, la velocidad de entrada $\left(v_{e}\right)$ a través de las aberturas no excederá cierto valor. Existen dos criterios para definir la velocidad $v_{e}$. El primer criterio es hidráulico y pretende que las pérdidas por fricción al entrar el agua al ademe no afecten la eficiencia del pozo; para esto se recomienda que la velocidad de entrada no exceda un valor entre 0.61 y 1.22 $\mathrm{m} / \mathrm{s}$ (Williams, 1985). El segundo criterio busca minimizar el potencial de corrosión y para esto se recomienda limitar la velocidad de entrada al ademe ranurado a un valor menor a $0.03 \mathrm{~m} / \mathrm{s}$ (Williams, 1981). Considerando que el área abierta por metro lineal de ademe instalado en el pozo SLT es de $1.417 \mathrm{~cm}^{2}$ (Figura 2), la velocidad de entrada al ademe durante el aforo 3 se muestra en la Tabla 3. Durante el aforo $3 v_{e}$ cumple con el límite sugerido por el criterio hidráulico pero rebasa el límite sugerido para evitar la corrosión. Sin embargo, para evaluar las condiciones para incrustación o corrosión se deben considerar las características químicas del agua y el potencial de óxido reducción, lo cual está fuera del ámbito de este trabajo.

\section{Discusión}

Los parámetros $K$ y $S_{s}$ estimados mediante inversión del aforo 3 corroboran los resultados obtenidos por Morales-Casique et al. (2014), mientras que el análisis de los errores de estimación demuestra su exactitud como parámetros efectivos definidos por las ecuaciones (10) y
(11). Cabe recalcar que adicional a los factores incluidos en la modelación de Morales-Casique et al. (2014), la inversión en este trabajo considera además pérdidas de carga lineales y el efecto del volumen de agua almacenado dentro del pozo. Más importante aún es el hecho de que los valores de $K$ y $S_{s}$ estimados son los primeros valores reportados en la literatura que son representativos de la profundidad entre 1140 y 2008 m en la Cuenca de México y son sustentados mediante una prueba hidráulica de larga duración (el aforo 3 tuvo una duración de más de 6 días). Estos valores proporcionan una referencia importante, a una escala de centenas de metros, como parámetros iniciales para modelos de flujo de agua subterránea a escala regional en la Cuenca de México.

Adicionalmente, los valores de los parámetros en la Tabla 1 constituyen valores de referencia para planear el aforo y realizar el diseño constructivo preliminar de nuevas captaciones a profundidades similares que el pozo SLT. Con base en un caudal de diseño (o deseado), la ecuación (6) con los parámetros de la Tabla 1 permiten obtener un estimado del abatimiento total esperado en el pozo y estimar la longitud de columna mínima necesaria para conducir el aforo (más la requerida para garantizar la sumergencia de la bomba). La longitud de la columna del equipo de aforo debe ser mayor al abatimiento esperado con el caudal de diseño para que el aforo incluya caudales mayores y realmente pueda proporcionar la información necesaria para seleccionar un caudal óptimo de operación del pozo (posiblemente mayor al caudal de diseño). En este contexto, cabe señalar un par de factores adicionales en la planeación de un aforo. En primer lugar, la bomba se debe seleccionar de manera que pueda proporcionar un caudal mayor al caudal de diseño bajo las condiciones de carga dinámica total esperada. En segundo lugar, el motor debe estar en condiciones de proveer la potencia necesaria para operar la bomba a las revoluciones necesarias para alcanzar los caudales deseados. La experiencia con los aforos 1 y 2 en el pozo SLT ilustran estos aspectos. Los caudales máximos alcanzados en los aforos 1 y 2 fueron de $38 \mathrm{~L} / \mathrm{s}$ y $47.4 \mathrm{~L} / \mathrm{s}$, respectivamente, mientras que durante la última etapa del aforo 3 se alcanzó un caudal sostenido de $62 \mathrm{~L} / \mathrm{s}$, lo que demostró la posibilidad de extraer un caudal mayor al sugerido por los aforos 1 y 2 .

\section{Conclusiones}

Conductividad hidráulica, almacenamiento específico y

Tabla 3. Velocidades de entrada al ademe ranurado durante el aforo 3.

\begin{tabular}{lcccccccccc}
\hline Etapa & 1 & 2 & 3 & 4 & 5 & 6 & 7 & 8 & 9 & 10 \\
\hline Caudal (1/s) & 12.27 & 18.03 & 21.16 & 27.98 & 32.93 & 39.19 & 47.21 & 55.43 & 62.16 & 70.5 \\
$v_{e}(\mathrm{~m} / \mathrm{s})$ & 0.11 & 0.17 & 0.2 & 0.26 & 0.31 & 0.37 & 0.44 & 0.52 & 0.58 & 0.66 \\
\hline
\end{tabular}


parámetros de pozo fueron estimados mediante inversión de una prueba de aforo (bombeo escalonado) en el pozo SLT. El modelo utilizado considera abatimiento debido al acuífero, pérdidas de carga lineales y no lineales debidas al pozo e incluye también el efecto del agua almacenada dentro de la perforación. Con base en estos parámetros, las pérdidas de carga debidas a la construcción del pozo fueron estimadas durante el desarrollo y durante dos aforos más. Los valores de conductividad hidráulica y almacenamiento específico constituyen los primeros valores reportados en la literatura para la profundidad entre 1176 y $2008 \mathrm{~m}$, que son representativos de una escala de cientos de $m$ y que pueden ser utilizados como parámetros iniciales en la calibración de modelos regionales de flujo de agua subterránea en la Cuenca de México. Adicionalmente, los parámetros del acuífero y del pozo SLT proporcionan valores de referencia útiles para planear aforos y el diseño constructivo de pozos de condiciones similares al pozo SLT.

Finalmente, los resultados evidencian el proceso de limpieza y desarrollo que ocurre en la vecindad de la perforación. También se ilustra de manera clara que alternar periodos de reposo y bombeo puede ser más efectivo para desarrollar el pozo que únicamente incrementar el caudal de extracción. En futuras exploraciones, es conveniente considerar medidas adicionales para hacer más eficiente el proceso de limpieza del pozo, tales como pistoneo e inyección de aire.

\section{Agradecimientos}

Este trabajo fue financiado mediante el convenio 0627-1O-ED-DT-1-11 con el Sistema de Aguas de la Ciudad de México. En particular se agradece a los ingenieros Ramón Aguirre Díaz, Fernando A. Ávila Luna y Alejandro Escobedo por las facilidades otorgadas. Los comentarios y sugerencias de Armando O. Hernández Valdés ayudaron a mejorar este trabajo.

\section{Referencias}

Arce, J.L., Layer, P.W., Morales-Casique, E., Benowitz, J.A., Rangel, E., Escolero, O., 2013, New constraints on the subsurface geology of the Mexico City Basin: The San Lorenzo Tezonco deep well, on the basis of ${ }^{40} \mathrm{Ar} /{ }^{39} \mathrm{Ar}$ geochronology and whole-rock chemistry: Journal of Volcanology and Geothermal Research, 266, 34-49.

Arce, J.L., Layer, P., Martínez, I., Salinas, J.I., Macías-Romo, M.C., Morales-Casique, E., Benowitz, J., Escolero, O., Lenhardt, N., 2015, Geología y estratigrafía del pozo profundo San Lorenzo Tezonco y de sus alrededores, sur de la Cuenca de México: Boletín de la Sociedad Geológica Mexicana, 67(2), 123-143.

Avci, C.B., 1992, Parameter-estimation for step-drawdown tests: Ground Water, 30(3), 338-342.

Avci, C.B., Ciftci, E., Sahin, A.U., 2010, Identification of aquifer and well parameters from step-drawdown tests: Hydrogeology Journal, 18(7), 1591-1601.

Barker, J.A., Herbert, R., 1992, A simple theory for estimating well losses: With application to test wells in Bangladesh: Hydrogeology Journal,
$0(0), 20-31$

Batu, V., 1998, Aquifer Hydraulics: A Comprehensive Guide to Hydrogeologic Data Analysis. New York: John Wiley \& Sons, Inc., $727 \mathrm{p}$.

Camacho-V., R.G., Vásquez-C., M., 1992, Comment on "Analytical solution incorporating nonlinear radial flow in confined aquifers" by Zekâi Sen: Water Resources Research, 28(12), 3337-3338.

Doherty, J., 2002, PEST Model-Independent Parameter Estimation, User Manual, 5th Edition, Brisbane, Australia: Watermark Numerical Computing, $336 \mathrm{p}$.

Forchheimer, P., 1901, Wasserbewegung durch Boden: Zeitschrift Verein Deutscher Ingenieure, 45, 1782-1788.

Jacob, C.E., 1947, Drawdown test to determine effective radius of artesian well: Transactions of the American Society of Civil Engineering, $112,1047-1064$

Jha, M.K., Kumar, A., Nanda, G., Bhatt, G., 2006, Evaluation of traditional and nontraditional optimization techniques for determining well parameters from step-drawdown test data: Journal of Hydrologic Engineering, 11(6), 617-630.

Kawecki, M.W., 1995, Meaningful interpretation of step-drawdown tests: Ground Water, 33(1), 23-32.

Kruseman, G.P., De Rider, N.A., 1994. Analysis and Evaluation of Pumping Test Data. Wageningen, The Netherlands: International Institute for Land Reclamation and Improvement, $377 \mathrm{p}$.

Louwyck, A., Vandenbohede, A., Lebbe, L., 2010, Numerical analysis of step-drawdown tests: Parameter identification and uncertainty: Journal of Hydrology, 380(1-2), 165-179.

Mathias, S.A., Butler, A.P., Zhan, H., 2008, Approximate solutions for Forchheimer flow to a well: Journal of Hydraulic Engineering, 134(9), 1318-1325.

Mathias, S.A., Todman, L.C., 2010, Step-drawdown tests and the Forchheimer equation: Water Resources Research, 46(W07514), DOI: 10.1029/2009WR008635.

Morales-Casique, E., Escolero, O.A., Arce, J.L., 2014, Resultados del pozo San Lorenzo Tezonco y sus implicaciones en el entendimiento de la hidrogeología regional de la cuenca de México: Revista Mexicana de Ciencias Geológicas, 31(1), 64-75.

Peña-Díaz, S.A., Arreguín-Mañón, J., 1989, Eficiencias electromecánicas de los equipos de bombeo y de las condiciones hidráulicas de los pozos: Ingeniería Hidráulica en México, 311, 17-24.

Pérez-Cruz, G.A., 1988, Estudio sismológico de reflexión del subsuelo de la ciudad de México, México, D.F., tesis de maestría, Facultad de Ingeniería, Universidad Nacional Autónoma de México, 83 p.

Pérez-Franco, D., 1994. El flujo no lineal hacia pozos de extracción en acuíferos de baja conductividad hidráulica. Boletín Geológico y Minero, 105(5), 454-460.

Pérez-Franco, D., 1996. Flujo no lineal impermanente hacia un pozo en un acuífero semiconfinado, en Fagundo, J., Pérez-Franco, D., García J.M., Álvarez-Nodarse, A., Morell, I. (eds.), Contribuciones a la Hidrogeología y Medio Ambiente en Cuba, Castelló de la Plana, España: Publicacions de la Universitat Jaume I, 123-135.

Rathod, K.S., Rushton, K.R., 1991, Interpretation of pumping from twozone layered aquifers using a numerical model: Ground Water, 29(4), 499-509.

Rorabaugh, M.I., 1953, Graphical and theoretical analysis of stepdrawdown tests of artesian well: Proceedings Separate No. 362 ASCE, 79, 1-23.

Rushton, K.R., 2003, Groundwater Hydrology: Conceptual and Computational Models. West Sussex, England: John Wiley \& Sons Ltd, $416 \mathrm{p}$.

Şen, Z., 1988, Analytical solution incorporating nonlinear radial flow in confined aquifers: Water Resources Research, 24(4), 601-606.

Shekhar, S., 2006, An approach to interpretation of step drawdown tests: Hydrogeology Journal, 14(6), 1018-1027.

Tinoco-Michel, J.A., 2007. Modelo matemático del acuífero profundo del valle de México usando diferencias finitas y superficies finitas integradas: simulación numérica con el programa Modflow, tesis de licenciatura, Universidad Michoacana de San Nicolás de Hidalgo, $73 \mathrm{p}$. 
Wen, Z., Huang, G., Zhan, H., Li, J., 2008, Two-region non-Darcian flow toward a well in a confined aquifer: Advances in Water Resources, 31(5), 818-827.

Williams, D.E., 1985, Modern techniques in well design: Journal / American Water Works Association, 77(9), 68-74.

Williams, E.B., 1981, Fundamental concepts in well design: Groundwater, 19(5), 527-542.
Manuscrito recibido: Septiembre 11, 2014

Manuscrito corregido recibido: Febrero 10, 2015

Manuscrito aceptado: Febrero 15, 2015 